\title{
Ultrafast vibrational motion of carbon nanotubes in different $\mathrm{pH}$ environments
}

\author{
Kotaro Makino, ${ }^{1}$ Atsushi Hirano, ${ }^{1}$ Kentaro Shiraki, ${ }^{1}$ Yutaka Maeda, ${ }^{2,3}$ and Muneaki Hase ${ }^{1,3, *}$ \\ ${ }^{1}$ Institute of Applied Physics, University of Tsukuba, 1-1-1 Tennodai, Tsukuba 305-8573, Japan \\ ${ }^{2}$ Department of Chemistry, Tokyo Gakugei University, 4-1-1 Nukuikitamachi, Koganei, Tokyo 184-8501, Japan \\ ${ }^{3}$ PRESTO-JST, 4-1-8 Honcho, Kawaguchi, Saitama 332-0012, Japan
}

(Received 2 June 2009; revised manuscript received 12 November 2009; published 21 December 2009)

\begin{abstract}
We have used a femtosecond pump-probe impulsive Raman technique to explore the ultrafast dynamics of micelle suspended single-walled carbon nanotubes (SWNTs) in various $\mathrm{pH}$ environments. The structures of coherent phonon spectra of the radial breathing modes exhibit significant $\mathrm{pH}$ dependence, to which we attribute the effect of the protonation at the surface of SWNTs, resulting in the modification of electronic properties of semiconductor SWNTs. Analysis of the time-domain data using a time-frequency transformation uncovers also a second transient longitudinal breathing mode, which vanishes after 1 ps of the photoexcitation.
\end{abstract}

DOI: $10.1103 /$ PhysRevB.80.245428

PACS number(s): 78.47.J-, 78.67.Ch, 63.22.Gh, 63.20.kd

Carbon nanotubes (CNTs) are one of the most intriguing nanomaterials, which are useful for molecular devices, such as a chemical sensor ${ }^{1}$ and a nanomachine capable for medical science. ${ }^{2}$ One potential way to realize the nanomachine by the use of CNTs, which can work in biological systems, e.g., a drug delivery system, is to use them in solution with proteins. ${ }^{2}$ Therefore, CNT devices will be used in various $\mathrm{pH}$ environments in biological systems, e.g., the typical $\mathrm{pH}$ of human arterial blood is $\approx 7.4$ that is weakly alkaline. Since the chemical reactivity of CNTs is dominated by the fundamental physical processes on their surface, investigation of dynamics at surfaces, such as charge transfer, ${ }^{3}$ excitonplasmon coupling, ${ }^{4}$ and electron-phonon energy transfer, ${ }^{5}$ is crucial to prepare suitable environments for CNT devices.

Single-walled carbon nanotubes (SWNTs) possess simplest structure of CNTs and it has metallic or semiconducting conductivity, depending on the arrangement of carbon atoms, referred as roll-up vectors. ${ }^{6}$ Recently frequency-domain spectroscopy has revealed the diameter-selective Raman scattering from radial breathing modes (RBMs) in SWNT, ${ }^{7,8}$ where the frequency of the RBMs depended on the inverse of the diameter. ${ }^{3}$ Moreover, Strano et al. ${ }^{9}$ have investigated the change in the Raman spectra of RBM induced by the protonation and showed that the protons change electronic structure of SWNT, i.e., protonation is band-gap selective and consequently the condition of resonant Raman scattering was modified, depending on the $\mathrm{pH}$ values. Coherent phonon spectroscopy is another potential method to study phonon dynamics in time-domain. Up to date, there have been only several studies on ultrafast phenomena in CNTs, ${ }^{10-14}$ and early time stage dynamics of photoexcitation of electronic system and subsequent phonon excitation and relaxation was not been well explored. Motivated by the observation of the early time stage dynamics of electron-phonon coupling, Lüer et al. ${ }^{15}$ have recently investigated the electron-phonon coupling by means of coherent phonon spectroscopy with $10 \mathrm{fs}$ time resolution, where they focused their attention to the high-frequency optical modes rather than the RBMs.

In this paper, the coherent oscillation of the RBMs in SWNTs has been measured in various $\mathrm{pH}$ environments to show that the subpicosecond relaxation dynamics as well as the coherent phonon spectra of the RBM are very sensitive to the change in the electronic structure of SWNT upon the protonation.

The samples of SWNTs were produced via the highpressure catalytic $\mathrm{CO}$ decomposition process. To disperse SWNTs in aqueous solution, we used sodium dodecyl sulfate (SDS). Solution containing $100 \mathrm{mM}$ SDS in distilled water was mixed with SWNT powder. The SWNTs were dispersed by ultrasonication for $30 \mathrm{~min}$ at $20^{\circ} \mathrm{C}$. Nondispersed SWNTs were removed by filtration of the solution using Whatman filter paper no. 41. Successively, they were diluted threefold into $50 \mathrm{mM}$ citrate-phosphate-borate buffer solutions at different pHs (3, 4, 5, 6, and 7) and they were filled in the 5-mm-thick quartz cells. Absorption spectra obtained for SWNTs in SDS solution (Fig. 1) showed that the sharp peaks corresponding to the exciton bands ${ }^{16}$ appeared on the background absorption, which was assigned to $\pi$-plasmon contribution due to graphitic carbon or nanotube bundles. ${ }^{17}$ As shown in the inset of Fig. 1, the absorbance at $820 \mathrm{~nm}$ due to exciton resonance gradually increased as the $\mathrm{pH}$ value increased.

Time-resolved transient transmission of the sample was measured by employing a fast scanning delay technique us-

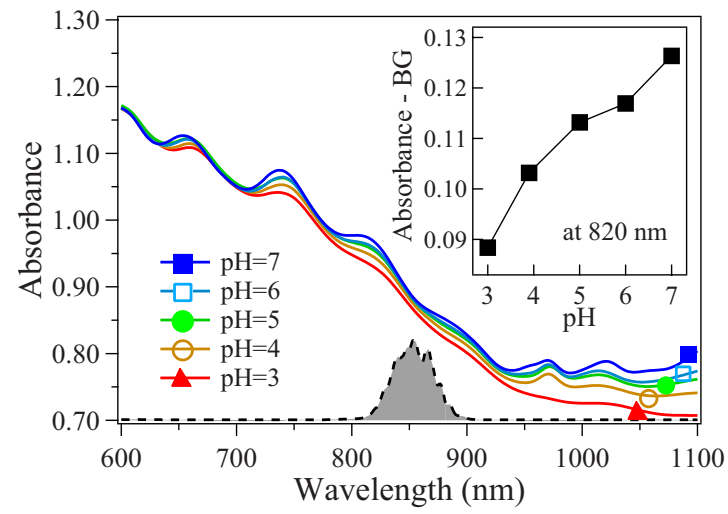

FIG. 1. (Color online) Absorption spectra obtained in SWNT/ SDS samples at various $\mathrm{pH}$ values. The dashed line with gray shading represents laser spectrum centered at $850 \mathrm{~nm}$, showing full bandwidth of $\sim 100 \mathrm{~nm}$. The inset shows the absorbance at $820 \mathrm{~nm}$ as the function of $\mathrm{pH}$ value obtained by subtracting background absorption based on $\pi$-plasmon model (see the text). 


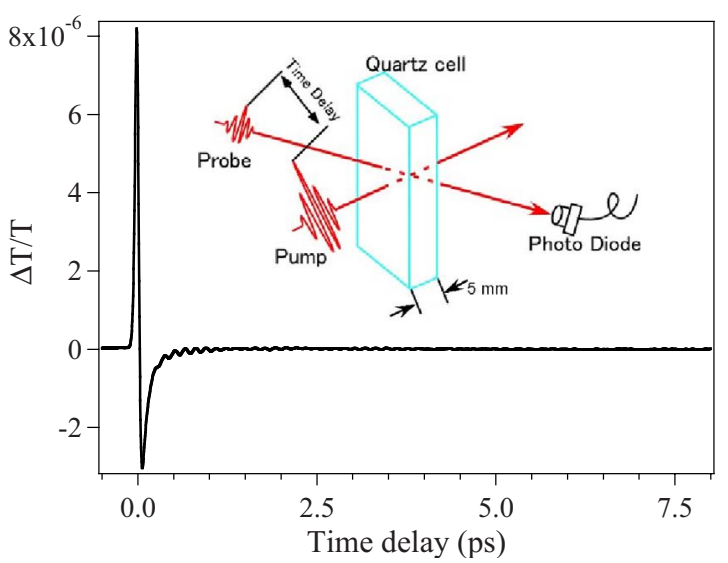

FIG. 2. (Color online) Time-resolved transmission for SWNT/ SDS sample at $\mathrm{pH}=3$. Coherent $\mathrm{RBMs}$ are observed just after the transient electronic response at $\tau=0$ ps. Pump-probe setup around the quartz cell is schematically shown in the inset.

ing a mode-locked Ti:sapphire laser. Nearly collinear, pump, and probe pulses [20 fs pulse duration; $850 \mathrm{~nm}(=1.46 \mathrm{eV})$ wavelength; and $80 \mathrm{MHz}$ repetition rate] were focused to a $70 \mu \mathrm{m}$ spot into the sample cell. The average power of the pump and probe beams were fixed at 40 and $3 \mathrm{~mW}$, respectively. The transient transmission $(\Delta T / T)$ was recorded as a function of the time delay $\tau$ between the pump and probe pulses.

Figure 2 shows $\Delta T / T$ signal observed for SDS suspended $\mathrm{SWNT}$ in solution at $\mathrm{pH}=3$. The transient electronic response appears at $\tau \approx 0$ ps and it is due to the change in the third-order nonlinear susceptibility $\chi^{(3)}$ from water solution ${ }^{11}$ as well as due to the excitation and subsequent relaxation of photoexcited carriers in SWNTs. The transient electronic response is followed by the coherent oscillations due to the RBMs, whose frequency $\omega_{\mathrm{RBM}}$ inversely depends on the diameter of SWNT by ${ }^{3}$

$$
\omega_{\mathrm{RBM}}=7.44[\mathrm{THz}] / R_{t}[\mathrm{~nm}],
$$

where $R_{t}$ is the diameter of SWNT. By subtracting the carrier responses from the time-domain data in Fig. $2{ }^{18}$ only the oscillatory components due to the coherent RBMs are obtained as shown in Fig. 3. The $\Delta T / T$ signal, thus obtained in the different $\mathrm{pH}$ environments $(\mathrm{pH}=3,4,5,6$, and 7$)$, indicate that the amplitude of the coherent RBMs seems to increase with increasing the $\mathrm{pH}$ value, implying that the appearance of the RBM is sensitive to the $\mathrm{pH}$ environment. The coherent RBMs are fit by the linear combination of the damped harmonic oscillations,

$$
\begin{aligned}
\frac{\Delta T}{T}= & A_{L} e^{-t / \tau_{L}} \cos \left[2 \pi\left(\nu_{L}+\alpha_{L} t\right) t+\varphi_{L}\right] \\
& +A_{H} e^{-t / \tau_{H}} \cos \left[2 \pi\left(\nu_{H}+\alpha_{H} t\right) t+\varphi_{H}\right],
\end{aligned}
$$

where $A_{L}\left(A_{H}\right), \nu_{L}\left(\nu_{H}\right), \tau_{L}\left(\tau_{H}\right), \alpha_{L}\left(\alpha_{H}\right)$, and $\varphi_{L}\left(\varphi_{H}\right)$ are the amplitude, the static frequency, the dephasing time, the frequency chirp, and the initial phase of the dominant coherent RBMs of the lower $\left(\nu_{L} \approx 6.4 \mathrm{THz}\right)$ and the higher $\left(\nu_{H}\right.$ $\approx 7.2 \mathrm{THz}$ ) modes as shown in the Fourier-transformed (FT)

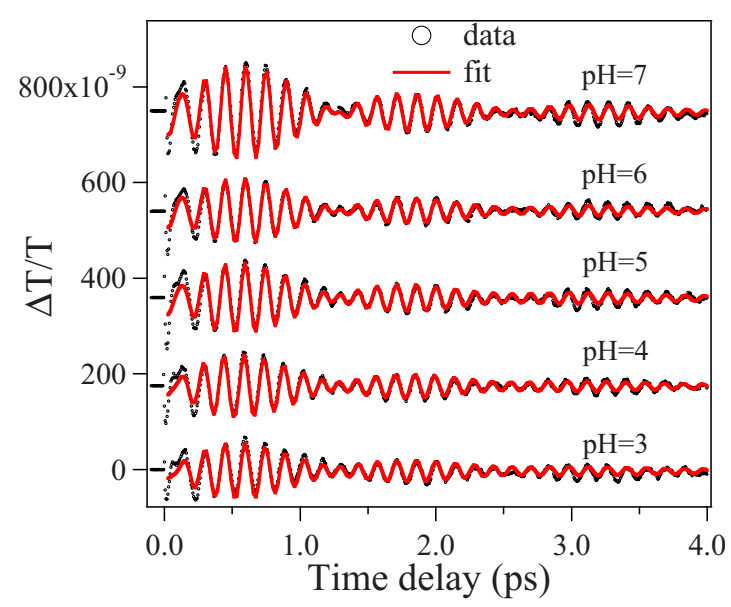

FIG. 3. (Color online) Time domain signal from the coherent RBM obtained by subtracting carrier responses from the timedomain transmission at various $\mathrm{pH}$ values. The solid lines represent the fit to the time-domain data with Eq. (2).

spectra in Fig. 4. Note that satellite peaks appear at the lower frequency side of the $6.4 \mathrm{THz}$ mode (Fig. 4), implying inhomogeneity of the tube diameters. ${ }^{8}$

By using the Kataura's plot it is possible to extract which chirality $(n, m)$ of SWNTs satisfies resonant condition for the Raman scattering and to identify the electronic state of SWNT, as semiconducting or metal. ${ }^{19}$ In our sample the diameter of SWNT has a range of $0.9-1.3 \mathrm{~nm}$. Therefore, both the dominant lower RBM at $6.4 \mathrm{THz}$ and the higher one at $7.2 \mathrm{THz}$ are considered to be the RBMs from the semiconductor SWNTs. In fact, according to Eq. (1), the peak of 6.4 $\mathrm{THz}$ corresponds to $\approx 1.16 \mathrm{~nm}$ tubes while the peak of 7.2 $\mathrm{THz}$ corresponds to $\approx 1.03 \mathrm{~nm}$ tubes, where the chirality of the lower mode can be $(13,2)$ and that of the higher mode can be $(12,1) .{ }^{20}$ Considering the photon energy used in the present study $(850 \mathrm{~nm})$, we can conclude that semiconducting SWNTs are excited through resonant impulsive stimu-

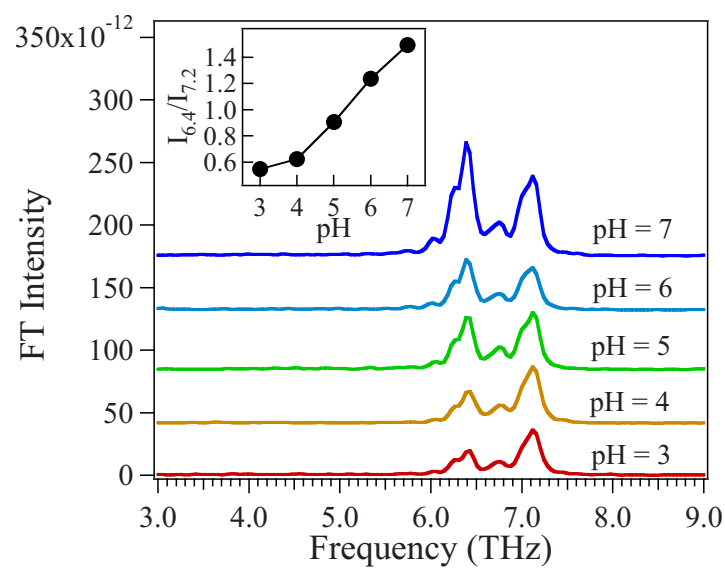

FIG. 4. (Color online) The FT spectra obtained from the timedomain signal for various $\mathrm{pH}$ values. The two dominant peaks appear at $6.4 \mathrm{THz}$ (lower mode) and at 7.2 THz (higher mode). The inset represents the ratio of the intensity of the lower mode to the higher mode, indicating the lower mode is enhanced in the order of $\mathrm{pH}=3,4,5,6$, and 7 . 
TABLE I. Main parameters obtained from the fittings of the oscillatory traces in Fig. 3 by using Eq. (2).

\begin{tabular}{ccccccc}
\hline \hline & $\begin{array}{c}\nu_{L} \\
\mathrm{pH}\end{array}$ & $\begin{array}{c}\nu_{H} \\
(\mathrm{THz})\end{array}$ & $\begin{array}{c}\tau_{L} \\
(\mathrm{ps})\end{array}$ & $\begin{array}{c}\tau_{H} \\
(\mathrm{ps})\end{array}$ & $\begin{array}{c}\alpha_{L} \\
\left(\mathrm{ps}^{-2}\right)\end{array}$ & $\begin{array}{c}\alpha_{H} \\
\left(\mathrm{ps}^{-2}\right)\end{array}$ \\
\hline 3 & $6.34 \pm 0.02$ & $7.18 \pm 0.02$ & $0.80 \pm 0.1$ & $1.97 \pm 0.1$ & $0.002 \pm 0.01$ & $-0.015 \pm 0.005$ \\
4 & $6.35 \pm 0.02$ & $7.19 \pm 0.02$ & $0.87 \pm 0.1$ & $1.95 \pm 0.1$ & $0.0002 \pm 0.01$ & $-0.018 \pm 0.005$ \\
5 & $6.33 \pm 0.02$ & $7.21 \pm 0.02$ & $0.85 \pm 0.1$ & $2.02 \pm 0.1$ & $0.002 \pm 0.01$ & $-0.024 \pm 0.005$ \\
6 & $6.34 \pm 0.02$ & $7.22 \pm 0.02$ & $0.94 \pm 0.1$ & $2.10 \pm 0.1$ & $0.002 \pm 0.01$ & $-0.027 \pm 0.005$ \\
7 & $6.32 \pm 0.02$ & $7.21 \pm 0.02$ & $1.08 \pm 0.1$ & $1.99 \pm 0.1$ & $0.006 \pm 0.01$ & $-0.024 \pm 0.005$ \\
\hline \hline
\end{tabular}

lated Raman process. ${ }^{21}$ Because of the broad bandwidth $(\sim 100 \mathrm{~nm}$ in full) of the laser used, the excitonic transitions around the critical point of $E_{22}$ (Ref. 6) are available at 800$900 \mathrm{~nm}$. Note that our laser can excite also transition corresponding to $E_{11}$ critical point beyond the gap. In this case, however, the intensity of the RBM would be negligibly small compared to those excited through the more resonant $E_{22}$ band.

We find in Fig. 3 that Eq. (2) fits the time-domain data well, although there is a significant discrepancy between the data and the fit at the early time stage, i.e., within the first oscillation cycle at $\leq 300$ fs. From the obtained fitting parameters (Table I) we notice that the static frequency of the coherent RBMs does not depend on the $\mathrm{pH}$ value, i.e., $\nu_{L}$ $=6.33 \pm 0.02 \mathrm{THz}$ and $\nu_{H}=7.20 \pm 0.02 \mathrm{THz}$, indicating that the static diameter of SWNTs is not effectively changed when the $\mathrm{pH}$ value is varied. On the other hand, the frequency chirp of the higher mode $\alpha_{H}$ is always negative, slightly depending on the $\mathrm{pH}$ value. This fact suggests that the dynamic frequency of the higher RBM is dependent on the time delay; the negative chirp means that the transient frequency is blueshifted at early time delays, followed by the redshift. Such the blueshift might be explained by the electron-phonon decoupling induced by the carrier doping, as observed for the $E_{2 g 2}$ mode in graphite ${ }^{22}$ and for the RBM in metallic SWNTs. ${ }^{23}$ Moreover, the dephasing time of the lower mode significantly increases with increasing the $\mathrm{pH}$ value; $\tau_{L}$ changes from 0.80 to $1.08 \mathrm{ps}$ while $\tau_{H}$ is almost unchanged as shown in Table I. Note that we cannot find any systematic change in the initial phase of the dominant coherent RBMs for the different $\mathrm{pH}$ values (not shown).

Figure 4 shows coherent phonon spectra obtained from the traces in Fig. 3 by using FT. It is found that the lower $\mathrm{RBM}$ at $6.4 \mathrm{THz}$ is strongly enhanced as the $\mathrm{pH}$ value increases, indicating that the lower protonation, the stronger intensity of the RBM at $6.4 \mathrm{THz}$. The effect of the protonation (and consequently the effect of decreasing the $\mathrm{pH}$ value) on the RBMs was in past examined by using Raman scattering, where the reduction in the Raman intensity of the semiconducting SWNTs was observed with increasing acidification (protonation) and the authors claimed that the acidification of the solution of surfactant-dispersed SWNT in water resulted in a reaction with protons at the sidewall of $\mathrm{CNT}^{9}$ Here, the protonation can be considered to capture free electrons from semiconducting SWNTs. Based on these arguments, $\mathrm{H}^{+}$ions would act as acceptors, changing the electronic property of semiconducting SWNT from $p$-type at
$\mathrm{pH}=3$ into nearly undoped at $\mathrm{pH}=7 .^{24}$ This may explain why the absorbance gradually increases as the $\mathrm{pH}$ value increases (Fig. 1) because of the reduced oscillator strength due to a partly emptied valence band. ${ }^{25}$ Note that the factor of the increase in the absorbance due to the exciton resonance $(\approx 1.43$ at $820 \mathrm{~nm}$ as shown in the inset of Fig. 1$)$ is smaller than the experimental observation $(\sim 4.0$ for the lower RBM as in Fig. 4), implying the existence of other contributions to the observed enhancement of the RBM amplitude in Fig. 4.

From Table I we found (i) the dephasing time of the lower $\mathrm{RBM}$ depends on the $\mathrm{pH}$ value while that of the higher RBM at $7.2 \mathrm{THz}$ is not dependent on $\mathrm{pH}$ and (ii) the dephasing time of the coherent RBM at $6.4 \mathrm{THz}$ is shorter than that of the higher RBM at 7.2 THz. Regarding to (i) above, the $\mathrm{pH}$ dependence of the dephasing time would be related to the carrier-phonon interaction at early time delays. In fact, Ostojic et al. ${ }^{24}$ reported that the early stage relaxation process of photoexcited carriers in SWNTs was found in similar time region, $0.3-1.2 \mathrm{ps}$, and the relaxation time depended on $\mathrm{pH}$ values; the lower $\mathrm{pH}$ value, the faster relaxation time (destroy the slow component). They also argued that based on the Burstein-Moss effect ${ }^{26}$ larger diameter tube (consequently lower frequency RBM) is more susceptible to this $\mathrm{pH}$ dependence because of the smaller accepter binding energies of larger diameter tubes. In addition to the effect described above, SWNT becomes $p$-type semiconductor as the $\mathrm{pH}$ value decreases. ${ }^{24}$ If this is the case, the lifetime of carriers is rather short at the lower $\mathrm{pH}$ values because of the shorter lifetime of the photogenerated holes ${ }^{27}$ and therefore the dephasing time of the coherent RBM, which is coupled with the photogenerated carriers, is also shorter. These considerations are consistent with the behavior of $\tau_{L}$ for the different $\mathrm{pHs}$ in Table I. Note that a possible reason why the dephasing time of the higher RBM is not dependent on $\mathrm{pH}$ is the electron-phonon decoupling, which would occur in a time scale of a few picoseconds ${ }^{22}$ and would stronger for the higher RBM. ${ }^{23}$

In the present study, RBMs are considered to be coupled with electrons through the optical deformation-potential interaction, ${ }^{28}$ i.e., the matrix element $\left|M_{e-p h}\right|$ of the electronphonon coupling can be described by $\left|M_{e-p h}\right|^{2}=\mid a / R_{t}^{2}$ $+\left.\left(\nu b / R_{t}\right) \cos 3 \theta\right|^{2}$, where $a$ and $b$ are the constants, $\nu[$ $=\bmod (n-m, 3)]$ is the chiral index, and $\theta$ is the chiral angle. $^{20,29}$ Thus the electron-phonon coupling strength decreases if the diameter of the SWNT $\left(R_{t}\right)$ increases. Consequently, regarding to the note (ii) above, the shorter dephasing time of the lower frequency RBM, corresponding to the 
larger diameter tubes, cannot be explained by the electronphonon coupling since the electron-phonon matrix element of the deformation-potential interaction becomes weak when the diameter of SWNT increases. ${ }^{28,29}$ Instead of considering electron-phonon coupling, inhomogeneous damping of the lower frequency RBM plausibly accounts for the shorter dephasing time of the lower RBM than that of the higher RBM. $^{30}$

From the considerations above, the reason why the FT intensity enhancement in Fig. 4 depends on the chirality may be explained by the increased dephasing time of the lower mode $\left(\tau_{L}\right)$, that is, a factor of $\tau_{L}(\mathrm{pH}=7) / \tau_{L}(\mathrm{pH}=3)=1.35$ from Table I. Since the amplitude $A$ of the coherent phonon would be proportional to $\gamma^{-1}(=\tau)$, where $\gamma$ is the damping, ${ }^{31}$ we expect the enhancement of the FT intensity $\left(A^{2}\right)$ for the lower RBM by a factor of 1.82 . This value is smaller than the experimental observation ( $\sim 4$ as in Fig. 4), however, the effect of the change in $\tau_{L}$ would dominantly contribute to the enhancement of the FT intensity.

In order to analyze ultrafast relaxation of the coherent RBM in detail, we first utilize the continuous wavelet transform (CWT) method $^{32}$ to obtain the time-frequency chronograms from the time-domain datum for $\mathrm{pH}=3$ presented in Fig. 3. Note that there was no considerable dependence of the CWT spectra on the $\mathrm{pH}$ value, excepting the ratio of the spectral intensities of the coherent RBMs. In Fig. 5(a) it is found that a coherent phonon response appears at $4-5 \mathrm{THz}$ at the early time delay of near $\tau=0 \mathrm{ps}$, followed by the strong peak at $6-8 \mathrm{THz}$ due to the coherent RBMs. This new mode at $\approx 4 \mathrm{THz}$ decay so fast $(\leq 1 \mathrm{ps}$, i.e., too broad in the frequency domain) that the FT analysis in Fig. 4 could not detect the mode. To gain more insight on the new mode at $\approx 4 \mathrm{THz}$, we use also a discrete Gabor transform (GT), ${ }^{33}$ which is expressed by the following equation:

$$
I(\omega, \Delta \tau)=\int_{0}^{\infty}\left(\frac{\Delta T}{T}\right) \exp \left[-\left(\frac{t-\Delta \tau}{\sigma}\right)^{2}\right] \exp (-i \omega t) d t .
$$

Here, $\Delta \tau$ and $\sigma(=1.0 \mathrm{ps})$ represent the time delay and the width of the window function, respectively. In this method, the window function (Gaussian) extracts oscillatory components at $\Delta \tau$ and thus GT method achieves time-resolved FT. ${ }^{34}$ As shown in Fig. 5(b), it is revealed that a broad peak at $\approx 4.4 \mathrm{THz}$ appears within $\approx 1 \mathrm{ps}$. This peak frequency is consistent with that of the new mode observed in CWT spectra in Fig. 5(a) and appears to be the longitudinal breathing (LB) mode of SWNT that is another coherent mode identi-
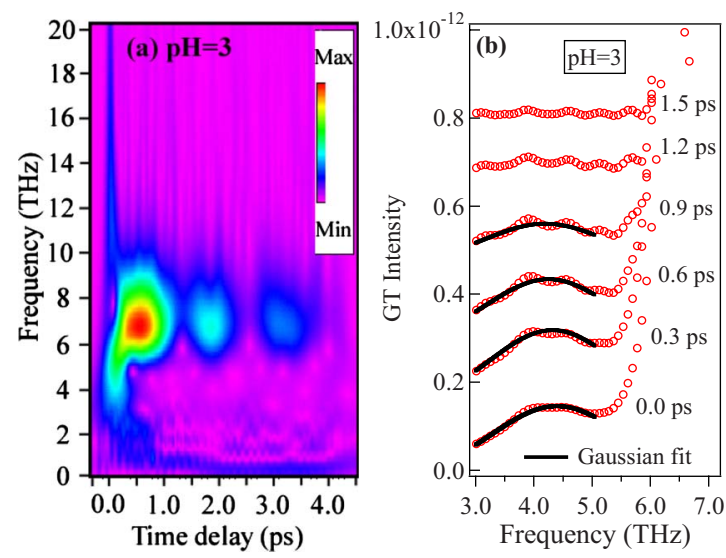

FIG. 5. (Color online) (a) The CWT chronogram for $\mathrm{pH}=3$. (b) Enlarged GT spectra around $4 \mathrm{THz}$ for $\mathrm{pH}=3$ in which the broadband appears at $\approx 4.4 \mathrm{THz}$. The solid curve in (b) is the fit to the GT spectra with a Gaussian function.

fied recently in molecular-dynamics simulations. ${ }^{35}$ This LB mode would thermalize fast due to inertial confinement along the tubular length. ${ }^{36}$

In conclusion, we have explored the ultrafast vibrational dynamics of coherent RBMs in real time using the femtosecond transmission technique based on impulsive Raman scattering. The two dominant RBMs were observed at 6.4 and 7.2 $\mathrm{THz}$, whose spectral intensities depended on the $\mathrm{pH}$ value. The enhancement of the lower RBM $(6.4 \mathrm{THz})$ at the higher $\mathrm{pH}$ values could be attributed to the change in the electronic structure of SWNT from $p$-doped to nondoped nature by the deprotonation effect. Dephasing time of the lower RBM extracted from the time-domain data exhibited $\mathrm{pH}$ dependence, which was explained by the electron-phonon coupling through deformation-potential interaction while that of the higher RBM did not depend on the $\mathrm{pH}$ value, implying existence of the electron-phonon decoupling in a few picoseconds. Analysis of the time-domain data by both the continuous wavelet transform and the Gabor transform methods revealed the new peak at $\approx 4.4 \mathrm{THz}$ in the transient response within $1 \mathrm{ps}$, which appeared to be the signature of the longitudinal breathing mode of SWNT.

The authors acknowledge T. Dumitrică for stimulating discussions and T. Kameda for his helpful comments on the coherent phonon spectra. This work was partly supported by the Step-up Support Program for the Young Scientist from University of Tsukuba.

\footnotetext{
*mhase@bk.tsukuba.ac.jp

${ }^{1}$ A. Modi, N. Koratkar, E. Lass, B. Q. Wei, and P. M. Ajayan, Nature (London) 424, 171 (2003).

${ }^{2}$ M. Zhang, T. Murakami, K. Ajima, K. Tsuchida, A. S. D. Sandanayaka, O. Ito, S. Iijima, and M. Yudasaka, Proc. Natl. Acad. Sci. U.S.A. 105, 14773 (2008).
}

${ }^{3}$ A. M. Rao, E. Richter, S. Bandow, B. Chase, P. C. Eklund, K. A. Williams, S. Fang, K. R. Subbaswamy, M. Menon, A. Thess, R. E. Smalley, G. Dresselhaus, and M. S. Dresselhaus, Science 275, 187 (1997).

${ }^{4}$ I. V. Bondarev, K. Tatur, and L. M. Woods, Opt. Commun. 282, 661 (2009). 
${ }^{5}$ T. Hertel and G. Moos, Phys. Rev. Lett. 84, 5002 (2000).

${ }^{6}$ R. Saito, G. Dresslhaus, and M. S. Dresselhaus, Physical Properties of Carbon Nanotubes (World Scientific, Singapore, 2003).

${ }^{7}$ A. Jorio, R. Saito, J. H. Hafner, C. M. Lieber, M. Hunter, T. McClure, G. Dresselhaus, and M. S. Dresselhaus, Phys. Rev. Lett. 86, 1118 (2001).

${ }^{8}$ M. S. Dresselhaus, G. Dresselhaus, R. Saito, and A. Jorio, Phys. Rep. 409, 47 (2005).

${ }^{9}$ M. S. Strano, C. B. Huffman, V. C. Moore, M. J. O’Connell, E. H. Hároz, J. Hubbard, M. Miller, K. Rialon, C. Kittrell, S. Ramesh, R. H. Hauge, and R. E. Smalley, J. Phys. Chem. B 107, 6979 (2003).

${ }^{10}$ T. Hertel, A. Hagen, V. Talalaev, K. Arnold, F. Hennrich, M. Kappes, S. Rosenthal, J. McBride, H. Ulbricht, and E. Flahaut, Nano Lett. 5, 511 (2005).

${ }^{11}$ Y.-S. Lim, K.-J. Yee, J.-H. Kim, E. H. Hároz, J. Shaver, J. Kono, S. K. Doorn, R. H. Hauge, and R. E. Smalley, Nano Lett. 6, 2696 (2006)

${ }^{12}$ A. Gambetta, C. Manzoni, E. Menna, M. Meneghetti, G. Cerullo, G. Lanzani, S. Tretiak, A. Piryatinski, A. Saxena, R. L. Martin, and A. R. Bishop, Nat. Phys. 2, 515 (2006).

${ }^{13}$ K. Kato, K. Ishioka, M. Kitajima, J. Tang, R. Saito, and H. Petek, Nano Lett. 8, 3102 (2008).

${ }^{14}$ J.-H. Kim, K.-J. Han, N.-J. Kim, K.-J. Yee, Y.-S. Lim, G. D. Sanders, C. J. Stanton, L. G. Booshehri, E. H. Hároz, and J. Kono, Phys. Rev. Lett. 102, 037402 (2009).

${ }^{15}$ L. Lüer, C. Gadermaier, J. Crochet, T. Hertel, D. Brida, and G. Lanzani, Phys. Rev. Lett. 102, 127401 (2009).

${ }^{16}$ F. Wang, G. Dukovic, L. E. Brus, and T. F. Heinz, Science 308, 838 (2005).

${ }^{17}$ B. J. Landi, H. J. Ruf, C. M. Evans, C. D. Cress, and R. P. Raffaelle, J. Phys. Chem. B 109, 9952 (2005).

${ }^{18}$ First, we fitted the time-domain data in Fig. 2 with a doubleexponential function for the time window of $[0, \infty]$. The residual of the fit showed satisfactory nice reproduction of the carrier response. After that, we subtracted the double-exponential function from the time-domain data. Thus, we have obtained only oscillatory parts of the time-domain signal (Fig. 3) for the time window of $[0, \infty]$.

${ }^{19}$ H. Kataura, Y. Kumazawa, Y. Maniwa, I. Umezu, S. Suzuki, Y. Ohtsuka, and Y. Achiba, Synth. Met. 103, 2555 (1999).
${ }^{20}$ S. V. Goupalov, B. C. Satishkumar, and S. K. Doorn, Phys. Rev. B 73, 115401 (2006).

${ }^{21}$ T. E. Stevens, J. Kuhl, and R. Merlin, Phys. Rev. B 65, 144304 (2002).

${ }^{22}$ K. Ishioka, M. Hase, M. Kitajima, L. Wirtz, A. Rubio, and H. Petek, Phys. Rev. B 77, 121402(R) (2008).

${ }^{23}$ H. Farhat, K. Sasaki, M. Kalbac, M. Hofmann, R. Saito, M. S. Dresselhaus, and J. Kong, Phys. Rev. Lett. 102, 126804 (2009).

${ }^{24}$ G. N. Ostojic, S. Zaric, J. Kono, M. S. Strano, V. C. Moore, R. H. Hauge, and R. E. Smalley, Phys. Rev. Lett. 92, 117402 (2004).

${ }^{25}$ K. K. Kim, J. J. Bae, H. K. Park, S. M. Kim, H.-Z. Geng, K. A. Park, H.-J. Shin, S.-M. Yoon, A. Benayad, J.-Y. Choi, and Y. H. Lee, J. Am. Chem. Soc. 130, 12757 (2008).

${ }^{26}$ E. Burstein, Phys. Rev. 93, 632 (1954).

${ }^{27}$ F. Ganikhanov, K. C. Burr, and C. L. Tang, Appl. Phys. Lett. 73, 64 (1998).

${ }^{28}$ J. Jiang, R. Saito, Ge. G. Samsonidze, S. G. Chou, A. Jorio, G. Dresselhaus, and M. S. Dresselhaus, Phys. Rev. B 72, 235408 (2005).

${ }^{29}$ Y. Yin, A. N. Vamivakas, A. G. Walsh, S. B. Cronin, M. S. Ünlü, B. B. Goldberg, and A. K. Swan, Phys. Rev. Lett. 98, 037404 (2007).

${ }^{30}$ In general, the damping (the inverse of the dephasing time) can be expressed by $\gamma_{\text {total }}=\gamma_{0}+\gamma_{\text {e-ph }}+\gamma_{\text {inhomo }}$, where $\gamma_{0}$ is the temperature-dependent anharmonic decay rate, $\gamma_{e-p h}$ is the electron-phonon damping, and $\gamma_{\text {inhomo }}$ is the inhomogeneous damping. When the $\mathrm{pH}$ value is varied $\gamma_{e-p h}$ is most sensitive while $\gamma_{\text {inhomo }}$ dominates the difference in the dephasing time between the lower and the higher RBMs.

${ }^{31}$ M. Hase, K. Mizoguchi, H. Harima, S. I. Nakashima, and K. Sakai, Phys. Rev. B 58, 5448 (1998).

${ }^{32}$ M. Hase, M. Kitajima, A. M. Constantinescu, and H. Petek, Nature (London) 426, 51 (2003).

${ }^{33}$ D. Gabor, J. Inst. Electr. Eng. (London) 93, 429 (1946).

${ }^{34}$ M. Hase, M. Kitajima, S. I. Nakashima, and K. Mizoguchi, Phys. Rev. Lett. 88, 067401 (2002).

${ }^{35}$ T. Dumitrică, M. E. Garcia, H. O. Jeschke, and B. I. Yakobson, Phys. Rev. B 74, 193406 (2006).

${ }^{36}$ H. O. Jeschke, A. H. Romero, M. E. Garcia, and A. Rubio, Phys. Rev. B 75, 125412 (2007). 